\title{
Die Familie im Gefüge der Solidargemeinschaften: Ein Ansatz soziologisch orientierter Rechtsvergleichung
}

\author{
Alexander Graser
}

Vorbemerkung

I. Die Rolle der Familie in sozialrechtsvergleichender Perspektive - ein Forschungsansatz

1. Sozialrecht, Gleichheit und Gemeinschaft

a) Sozialrecht als gleichheitsgenerierendes Recht 374

b) Sozialrecht als ,egalitärer Arm der Aufklärung“

c) Die (Bezugs-)Gemeinschaft als Prämisse der Gleichheitsgenerierung 376

2. Die Familie im Sozialrecht

a) Die Familie: Bezugsgemeinschaft oder Hindernis kollektiver Gleichheitsgenerierung?

b) Der Rückzug der Familie als Solidargemeinschaft im positiven Recht

c) Die bleibende Bedeutung der Familie als Solidargemeinschaft

3. Die Familie als Solidargemeinschaft in rechtsvergleichender Perspektive $\quad 379$

a) Die Familie im US-amerikanischen Recht 379

b) Gegenüberstellung von US-amerikanischem und deutschem Recht $\quad 380$

II. Erweiterungen des Forschungsansatzes 381

1. Das erweiterte Gefüge rechtlich verfestigter Solidargemeinschaften 381

a) Illustration des erweiterten Gefüges rechtlich verfestigter

Solidargemeinschaften

b) Die Bedeutung des erweiterten Gefüges für den zuvor vorgestellten Ansatz

2. Die Familie als ein (auch) gewillkürtes Verhältnis 384

3. Die Bedeutung sozialer Dienste 385

III. Dogmatik und Sozialrechtsvergleich $\quad 386$

1. Rechtswissenschaftliche Erkenntnisziele $\quad 386$

2. Rechtsvergleichende Erkenntnisziele 387

3. Zur (verborgenen) Bedeutung der Dogmatik in der (Sozial-)
Rechtsvergleichung

a) Dogmatische Ziele der Rechtsvergleichung 388

b) Die unverzichtbare (Hilfs-)Funktion der Dogmatik 389

c) Dogmatische Expertise als Proprium der Sozialrechtsvergleichung $\quad 390$ 


\section{Vorbemerkung}

Der vorliegende Text ist eine erweiterte Fassung des Beitrags, den ich auf der Tagung vorgetragen habe, die in diesem Band dokumentiert wird. Das Ziel des Beitrags ist, einen Ansatz vorzustellen, mit dem die Rolle der Familie sozialrechtsvergleichend untersucht werden könnte. Den Ausgangspunkt bildet ein funktionales Verständnis des Sozialrechts, wie ich es von Hans F. Zacher übernommen habe und wie es sich besonders eignen sollte für einen funktionalen Sozialrechtsvergleich in der Tradition der grundlegenden Arbeiten des Jubilars und des von ihm begründeten Instituts.

Im Folgenden ist die ursprüngliche Gedankenführung des Vortrags zunächst beibehalten (I). Unweigerlich enthielt diese Fassung eine Reihe von Verkürzungen. Deswegen soll die Perspektive hier in einem zweiten Schritt um einige zentrale Aspekte erweitert werden, die teils auch in der Diskussion des Vortrags eine Rolle gespielt haben (II). Der allgemeinen Zielsetzung der Tagung entsprechend, schließt der Beitrag mit einigen methodischen Reflektionen zum hier vorgestellten Ansatz und den Möglichkeiten der Rechtsvergleichung (III).

\section{Die Rolle der Familie in sozialrechtsvergleichender Perspektive - ein Forschungsansatz}

Das Wesen des Sozialrechts, so hat Zacher es wiederholt formuliert, liege darin, dass es auf die Negation, auf den Ausgleich von (materieller) Ungleichheit gerichtet sei. Diesen Gedanken möchte ich im Folgenden zunächst thesenartig entfalten, ihn dann auf das Rahmenthema der Familie im Sozialrecht beziehen und abschließend einen kurzen rechtsvergleichenden Blick in die USA werfen.

\section{Sozialrecht, Gleichheit und Gemeinschaft}

So griffig die Formel vom Sozialrecht als einem Ungleichheiten ausgleichenden Recht auch sein mag, bedarf sie doch der Erläuterung: Was für Ungleichheiten sind gemeint? Und, vielleicht nicht ganz so nahe liegend, aber nicht minder zentral: Um wessen Ungleichheit geht es?

\section{a) Sozialrecht als gleichheitsgenerierendes Recht}

Ist Sozialrecht also gleichheitsgenerierendes Recht, so kann damit nicht gemeint sein, dass es alle Ungleichheiten restlos beseitigen könnte oder auch nur sollte. Viel- 
mehr widmet sich das Sozialrecht stets nur ganz bestimmten Ungleichheiten, und meist beseitigt es diese auch nicht, sondern lindert sie nur.

Da sind zum einen Ungleichheiten, die durch bestimmte Wechselfälle des Lebens entstehen. Ist die Erwerbsfähigkeit beispielsweise wegen Krankheit, aufgrund eines Unfalls oder im Alter reduziert, so greifen typischer Weise die entsprechenden Ausgleichsysteme der Sozialversicherung. Daneben dienen zum anderen die Systeme der sozialen Fürsorge und Förderung dazu, Armut zu mildern und Teilhabe zu befördern, etwa im Fall der Eingliederungshilfe oder auch der Leistungen der Ausbildungsförderung. Spiegelbildlich dazu werden ferner mit der Finanzierung all dessen die Einzelnen zwar regelmäßig in Abhängigkeit von ihrer individuellen Leistungsfähigkeit belastet, aber doch grundsätzlich nicht mit dem Effekt einer völligen Einebnung materieller Unterschiede.

Schließlich soll in einer eben nicht allein egalitären, sondern zugleich liberalen Ordnung auch Leistung sich lohnen, also in Vorteile und damit Ungleichheit übersetzen lassen. Was korrigiert wird, sind nur solche Ungleichheiten, die als zu weitreichend empfunden werden. Das gilt insbesondere dann, wenn sie als zufällig erscheinen, also nicht auf bewusste und steuerbare Handlungen oder Entscheidungen des Einzelnen zurückführbar sind.

\section{b) Sozialrecht als ,,egalitärer Arm der Aufklärung“}

In diesem Sinne lässt sich das Sozialrecht in Verbindung bringen mit einer Beobachtung, die der amerikanische Soziologe Talcott Parsons vor rund vier Jahrzehnten angestellt hat. ${ }^{1}$ Die Entwicklung moderner Gesellschaften sei, so Parsons, gekennzeichnet davon, dass zur Legimitation von Ungleichheiten immer weniger rekurriert werden könne auf das, was er ,askriptive Merkmale“ nannte - Merkmale also, die dem Einzelnen in dem Sinne von außen zugeschrieben werden, dass er sie selbst nicht beeinflussen kann.

Übersetzt in unseren Zusammenhang könnte man vielleicht wieder schlicht von „,Zufälligkeiten" sprechen, deren bestimmenden Einfluss auf unser Schicksal wir in kollektiver Ausübung unserer Autonomie und mit Mitteln des Sozialrechts zurückdrängen. Mit reichlich Pathos, aber durchaus in Parsons Sinne, könnte man in dieser Perspektive das Sozialrecht den „egalitären Arm der Aufklärung“ nennen. (Dass sie noch einen zweiten, den liberalen Arm hat, wurde ja bereits erwähnt, und auch deren Verschränkung ist hinlänglich bekannt.)

1 Parsons, Equality and Inequality in Modern Society, or: Social Stratification Revisited, in: Sociological Inquiry 40 (1970), S. $13 \mathrm{ff}$. 


\section{c) Die (Bezugs-)Gemeinschaft als Prämisse der Gleichheitsgenerierung}

In diesem Verständnis als gleichheitsgenerierendes Recht setzt Sozialrecht einen Bezugspunkt voraus. Es muss schließlich klar sein, wer mit wem verglichen wird. Wenn man von gleichheitsgenerierendem Recht spricht, bedarf es deswegen notwendig einer Bezugsgemeinschaft.

Die Bestimmung dieser Bezugsgemeinschaft dagegen folgt keiner logischen Notwendigkeit. Vielmehr befinden wir uns hier im Bereich soziologischer Deskription oder auch philosophischer Präskription. Dabei ist das Sozialrecht, wie wir es kennen, meist staatliches Recht. Nicht zufällig handelte es sich auch bei allen zuvor genannten Beispielen $^{2}$ durchweg um staatliche Regelungskomplexe. Die Bezugsgemeinschaft wäre insofern also typischer Weise die Nation - oder genauer: zumeist die Wohnbevölkerung des jeweiligen Staates.

Soweit zunächst die Deskription. In präskriptiver Perspektive dagegen erscheint manchem der Staat als zu klein. Und in der Tat lässt sich für eine universalistische Ethik nur schwer rechtfertigen, warum gleichheitsgerichtete Ambitionen an Landesgrenzen halt machen sollten. Kinderarmut zum Beispiel würde wohl in den Augen der meisten nach Abhilfe verlangen, und zwar unabhängig vom Ort dieser Armut und vom Aufenthalt derer, die helfen könnten. In der Annahme solch universeller Gleichheitsverpflichtung hat die Forderung nach einem „Weltsozialrecht" ihre Begründung - eine Utopie, die in Zachers Arbeit bekanntlich seit langem eine wichtige Rolle spielt.

\section{Die Familie im Sozialrecht}

Der konzeptionelle Hintergrund meiner Überlegungen wäre damit umrissen. Wie lässt sich nun die Rolle der Familie innerhalb eines so verstandenen Sozialrechts auffassen?

\section{a) Die Familie: Bezugsgemeinschaft oder Hindernis kollektiver}

\section{Gleichheitsgenerierung?}

Zunächst einmal ist deutlich, dass auch die Familie begriffen werden kann als eine Bezugsgemeinschaft für das Bestreben, solche Ungleichheiten auszugleichen, die als zufällig und nicht gerechtfertigt empfunden werden. Auch und vielleicht ganz besonders in dieser Gemeinschaft gilt schließlich das Prinzip des Füreinander-Einstehens. Dem entspricht die rechtliche Konzeption der Familie als Bedarfs- und Unterhaltsverband,

2 Vgl. oben I. 1. a. 
über deren konkrete Ausprägungen in den beiden vorangegangenen Beiträgen ${ }^{3}$ bereits einiges gesagt wurde.

Hier möchte ich mich deswegen zunächst auf die umgekehrte Perspektive konzentrieren. Für einen Moment sollen die Familie und ihr rechtlicher Schutz nicht als Medium der kollektiven Gleichheitsgenerierung betrachtet werden, sondern im Gegenteil als Ausnahme oder gar als Hindernis für diese Ambition. Denn was könnte zufälliger sein askriptiv mit Parsons Wort - als die Geburt? Schon die nationale Zugehörigkeit ist offensichtlich diesem Einwand ausgesetzt. Aber er wird umso stärker, je kleiner der Bezugsrahmen möglichen Ausgleichs ist. Und in der Tat ist die Familie als Bezugsgemeinschaft für die Förderung von Gleichheit sehr klein. Sie fällt in dieser Hinsicht weit zurück hinter dem Staat als der primären Bezugsgemeinschaft modernen Sozialrechts, und noch weiter hinter den größeren Gemeinschaften, wie sie universalistischen Utopien entsprächen. Der gängige Verweis auf die Subsidiarität staatlicher Ausgleichssysteme und auf den Primat familialer Einstandspflichten wirkt vor diesem Hintergrund wie ein anti-egalitäres Relikt aus prämodernen Zeiten. Und die Familie erscheint, so betrachtet, geradezu als Antipode eines im Wortsinne „modernen“ Sozialrechts.

\section{b) Der Rückzug der Familie als Solidargemeinschaft im positiven Recht}

Zugegebenermaßen ist dieses Porträt der Familie überzeichnet. Aber es hat durchaus einen realen Anhalt im positiven Recht. Denn die Zufälligkeit familialer Zugehörigkeit hat tatsächlich immer weiter an Bedeutung verloren für das Ausmaß sowohl sozialer Berechtigung als auch sozialer Verpflichtung.

Die großen kollektiven Sicherungssysteme bewirken ja nichts anderes, als dass sie die Verantwortung für Krankheit, Alter und andere Wechselfälle immerhin teilweise von den Schultern der Angehörigen der Betroffenen nehmen und sie weiter kollektivieren. ${ }^{4}$ Und es gilt ferner nicht nur im Bereich der Sozialversicherung, sondern auch auf Ebene des Unterhaltsrechts, dass sich entsprechende Tendenzen ausmachen lassen. So hat der BGH insbesondere die Verpflichtung, Unterhalt für die Eltern leisten, in den letzten Jahren Schritt für Schritt reduziert. ${ }^{5}$ Flankiert wurde diese Entwicklung bekannt-

3 Vgl. Eichenhofer, Sozialleistung und Familie, S. 353 ff. und Köbl, Sozialstaatsentlastung durch mehr Familiensolidarität, S. 393 ff., in diesem Band.

4 Allgemein zum Prozess der „Verdrängung des Familienrechts durch das Sozialrecht“ von Maydell, Grundlegung: Das Sozialrecht und seine Stellung im Gesamtsystem, in: Ders./Ruland/Becker (Hrsg.), Sozialrechtshandbuch, 4. Aufl. Baden-Baden 2008, § 1 Rdnr. 47 ff.

5 Meist geht es dabei um Konstellationen, in denen über die Zulässigkeit eines Rückgriffs durch den Sozialhilfeträger gestritten wird. Einen Überblick zu den elternunterhaltsbegrenzenden Entwicklungen in der Rechtsprechung vermittelt etwa Brudermüller, Elternunterhalt, NJW 2004, S. 633 ff.; diese Entwicklung darstellend und - wenngleich in relativ pauschaler Form - bestätigend auch die Entscheidung des BVerfG, 1 BvR 1508/96, vom 7.6.2005, insbesondere Absatz-Nr. 5. Eine kritische Erörterung findet sich bei Boecken/Klattenhoff, Der Elternunterhalt bei Pflegebedürftigkeit zwischen sozialstaatlicher Verantwortung und individueller Einstandspflicht, JZ 2006, S. 285 ff., 
lich vom Grundsicherungsgesetz, das in entsprechenden Konstellationen den Regress des Sozialhilfeträgers weitgehend ausschließt. ${ }^{6}$ Wenn ich also den Solidarverband Familie zuvor als anti-egalitären Anachronismus porträtiert habe, so war das zumindest nicht völlig aus der Luft gegriffen, sondern findet seine Entsprechung in den genannten Tendenzen im positiven Recht.

\section{c) Die bleibende Bedeutung der Familie als Solidargemeinschaft}

Andererseits ist dies aber, wie gesagt, nur die halbe Wahrheit - und womöglich sogar noch weniger. Denn dass der Staat die Familie als Solidarverband vollends ablösen würde, steht nicht zur Debatte. Er könnte dies nicht, weil er ihre Funktionen gerade hinsichtlich des unmittelbaren Füreinander-Sorgens nicht voll zu übernehmen vermöchte. In Deutschland wäre es ihm auch von Verfassungs wegen versagt. ${ }^{7}$ Darüber hinaus bedarf staatlich organisierte Solidarität - jedenfalls in ihrer gegenwärtigen Form - auch zu ihrem eigenen Funktionieren der Familie als Grundlage. Die vielfältigen Formen, in denen das Sozialrecht an die Familie knüpft, brauchen an dieser Stelle nicht eigens wiederholt zu werden. 8

Kurz: Ohne die Solidarität im Kleinen geht es nicht. Und man wird ferner annehmen können, dass auch diese Solidarität nicht ohne rechtliche Verfestigung auskommt. Offenkundig entspricht dies der modernen Praxis weitreichend verrechtlichter familialer Beziehungen. Aber es wäre anders auch kaum denkbar. Wenn denn diese Formen wechselseitiger Verpflichtung innerhalb der Familie einen verlässlichen Teil eines umfassenden Systems der Absicherung gegen die Wechselfälle des Lebens bilden sollen, so dürfte an ihrer Verrechtlichung kaum ein Weg vorbei führen.

Für das Sozialrecht als gleichheitsgenerierendes Recht folgt aus den vorangegangenen Überlegungen, dass es das Neben- und Ineinander unterschiedlicher Bezugsgemeinschaften zu organisieren hat. Diese Regelungsaufgabe hat das Recht fortwährend zu bewältigen, unter immer neuen sozialen Bedingungen ${ }^{9}$ und im zuvor skizzierten Span-

die betonen, dass die Inanspruchnahme erwachsener Kinder durch den Sozialhilfeträger keineswegs generell eine unbillige Härte darstelle und im Hinblick auf den typischer Weise zuvor geleisteten Unterhalt der Eltern an die Kinder grundsätzlich als legitimiert gelten solle.

6 Vgl. BGB1. 2001 I, S. 1310 (1335).

7 Vgl. Art. 6 I GG.

8 Vgl. dazu neben den vorangegangenen Beiträgen (Fußn. 3) im gegebenen Zusammenhang vor allem auch den Begleitbeitrag zum 64. Deutschen Juristentag von Fuchs, Empfiehlt es sich, die rechtliche Ordnung finanzieller Solidarität zwischen Verwandten im Unterhalt-, Pflichtteils-, Sozialhilfe- und Sozialversicherungsrecht neu zu gestalten?, JZ 2002, S. 785 ff.; aus jüngerer Zeit auch Felix, Die Familie zwischen Privatrecht, Sozialrecht und Steuerrecht, in: Mellinghoff (Hrsg.), Steuern im Sozialstaat, Köln 2006, S. 149 ff.

9 Für eine prägnante empirische Darstellung vieler Facetten dieses Wandels vgl. Lüscher, Widersprüchliche Mannigfaltigkeit: Ehe, Familie und Verwandtschaft im aktuellen gesellschaftlichen und 
nungsfeld von einerseits universell-egalitären Zielsetzungen und andererseits dem Bedürfnis, bestehende Formen kleinräumiger Solidarität zu festigen. Neu ist diese Herausforderung also nicht - vielleicht aber die Perspektive, jedenfalls soweit sie zum Ausgangspunkt einer sozialrechtsvergleichenden Untersuchung gemacht werden sollte.

\section{Die Familie als Solidargemeinschaft in rechtsvergleichender Perspektive}

Die Rolle der Familie im In- und Nebeneinander unterschiedlicher Solidargemeinschaften ist, soweit ich es überblicke, tatsächlich noch nicht zum Gegenstand einer (sozial-)rechtsvergleichenden Studie gemacht worden. Dabei verspräche dies durchaus interessante Erträge, wie im Folgenden illustriert werden soll. Freilich kann an dieser Stelle allenfalls eine grobe Skizze dessen gezeichnet werden, was in einem solchen Vergleich zu untersuchen wäre. Ihn auch durchzuführen, würde deutlich mehr Raum erfordern. Damit aber die möglichen Ergebnisse dennoch auch in dieser Kürze hervortreten, wurde für die Gegenüberstellung bewusst ein Land gewählt, dessen Recht in der hier interessierenden Frage einen starken Kontrast zur deutschen Rechtslage abgibt.

\section{a) Die Familie im US-amerikanischen Recht}

Die Familie als Adressatin staatlicher Sozialleistungen gibt es in den USA kaum. Nur im Bereich der ohnehin spartanischen Mindestsicherung gibt es derartige Leistungen. ${ }^{10}$ Familienförderung oder -lastenausgleich dagegen findet man dort nicht. Auch Unterhaltspflichten sind in den USA typischer Weise deutlich schwächer ausgeprägt als hierzulande. Die elterlichen Pflichten gegenüber den Kindern enden meist früher ${ }^{11}$ und

erbrechtlichen Kontext heute, ZEV 2004, S. 2 ff.; speziell zum Elternunterhalt derselbe/Hoch, Der Elternunterhalt: Pragmatisch akzeptiertes Recht, FRP 2003, S. 648 ff.

10 Bedeutsam sind insofern vor allem zwei bundesweite Programme: der ,Earned Income Tax CreditEITC“, eine Form der negativen Einkommenssteuer, die gerade Familien mit minderjährigen Kindern besondere Unterstützung gewährt, sowie die ,Temporary Assistance for Needy Families TANF“, eine Geldleistung für Familien mit eingeschränkter Erwerbsfähigkeit und minderjährigen Kindern. Für Einzelheiten zu diesen beiden zentralen und weiteren einschlägigen Leistungen vgl. den konzisen Überblick aus jüngerer Zeit von Finegold, The United States Federalism and its Counter-Factuals, in: Obinger/Leibfried/Castles (Hrsg.), Federalism and the Welfare State, Cambridge 2005, S. 138 ff., insbesondere S. 152 ff.; ausführlicher und in deutscher Sprache auch Graser, Dezentrale Wohlfahrtsstaatlichkeit im föderalen Binnenmarkt, Berlin 2001.

11 Vgl. Martiny, Unterhaltsrang und Unterhaltsrückgriff - Mehrpersonenverhältnisse und Rückgriffsansprüche im Unterhaltsrecht Deutschlands, Österreichs, der Schweiz, Frankreichs, Englands und der Vereinigten Staaten von Amerika, Tübingen 2000, Bd. I, S. 57 f., 244 ff. (zum Ausbildungsunterhalt Bd. II, S. 1293 ff.), sowie Dose, Auslandsberührung, in: Wendl/Staudigl, Das Unterhaltsrecht in der familienrichterlichen Praxis, 7. Aufl. München 2008, § 9 Rdnr. 220 ff. 
auch in umgekehrter Richtung fallen die Pflichten der Kinder gegenüber den Eltern geringer aus. ${ }^{12}$

Ein ähnliches Bild bietet ferner das US-amerikanische Erbrecht, das kaum Beschränkungen der Testierfreiheit nach Muster des deutschen Pflichtteilsrechts kennt. ${ }^{13}$ Die familiale Solidarität, so lässt sich zusammenfassen, erfährt insofern deutlich weniger rechtliche Verfestigung als bei uns. Durchaus stimmig erscheint vor diesem Hintergrund überdies, dass in den USA traditionell auch die Erbschaftssteuer für Familienangehörige weit über dem hiesigen Niveau liegt. 14

\section{b) Gegenüberstellung von US-amerikanischem und deutschem Recht}

Im Vergleich mit Deutschland erscheint das US-amerikanische Recht demnach als nahezu familienblind - und zwar auf beiden Augen, bei den Rechten wie auch bei den Pflichten. Im Nebeneinander der verschiedenen Bezugsgemeinschaften gleichheitsgenerierenden Rechts spielt in den USA die Familie eine deutlich geringere Rolle als bei uns. Der US-amerikanische Egalitarismus, so wäre vielleicht zu resümieren, ist universeller, weil er sich stärker an die größere Bezugsgemeinschaft hält, also an den Staat. Und er ist zugleich individualistischer, weil er den Einzelnen stärker ins Zentrum rückt und die Familie in den Hintergrund.

Das deutsche Recht mit seiner zwar vielleicht rückläufigen, aber noch immer starken Betonung der Familie als Solidargemeinschaft neben dem Staat erschiene demgegenüber zunächst in einem schlechten Licht: Es erschiene als partikularistisch und im zuvor spezifizierten Sinne als ,unmodern“. Aber ich hatte ja bereits gesagt, dass diese Bewertung einseitig ist und womöglich zu kurz greift. Und auch das illustriert der Kontrast zu den USA. Denn bekanntlich ist das auf materielle Gleichheit zielende Recht dort nicht nur in Bezug auf die Familie, sondern auch in Bezug auf die staatliche Gemeinschaft geringer ausgeprägt als in Deutschland.

Es liegt nahe, hier einen Zusammenhang zu vermuten. Denkbar wäre, dass dieser Befund schlicht Ausdruck einer generell geringeren Anerkennung egalitärer Werte oder Ziele in den USA ist. Es könnte aber auch ein Beleg dafür sein, dass rechtliche Ausprä-

12 Vgl. Martiny, Unterhaltsrang und Unterhaltsrückgriff (Fußn. 11), S. 252 f.; auch Dose, in: Wendl/Staudigl (Fußn. 11), Rdnr. 219. Einen Überblick über die Vielzahl der Regelungen des Bundesrechts sowie des Rechts ausgewählter US-Staaten gibt Rieck (Hrsg.), Ausländisches Familienrecht, 3. Aufl. München 2007.

13 Näher dazu in historischer und vergleichender Perspektive Beckert, Inherited Wealth, Princeton 2008, S. $69 \mathrm{ff}$.

14 Vgl. auch dazu Beckert, Inherited Wealth (Fußn. 13), S. 167 ff., insbesondere S. 193. Die Betonung liegt freilich auf ,traditionell“. Denn zu den sozialpolitisch bedenklichen Hinterlassenschaften des scheidenden Präsidenten George W. Bush zählt unter anderem ein Gesetz, nach dem diese Steuer im Jahr 2010 schlicht wegfallen soll; vgl. näher auch dazu Beckert, Inherited Wealth (Fußn. 13), S. 193 ff. 
gungen von Solidarität in unterschiedlichen Bezugsgemeinschaften einander nicht hindern, sondern im Gegenteil wechselseitig fördern. Die kleinräumige, familiale Solidarität erschiene in dieser Perspektive nicht als Konkurrentin, sondern ganz im Gegenteil als Nährboden für die Solidarität im größeren, staatlichen Rahmen.

Zugegebenermaßen weisen derlei Hypothesen über das hinaus, was eine juristische Betrachtung je überprüfen könnte. Spätestens hier ist der Rechtsvergleich an der Grenze angelangt, jenseits derer das Terrain der Soziologie beginnt. Einen Abweg freilich bedeutet dies nicht. Im Gegenteil ist dieses Überschreiten disziplinärer Grenzen, wie später noch auszuführen sein wird, für die Rechtvergleichung sogar der Normalfall. ${ }^{15}$

\section{Erweiterungen des Forschungsansatzes}

Die vorangegangenen Überlegungen sind in mancher Hinsicht verkürzt. Wollte man den Ansatz weiter verfolgen und ihn im Rahmen einer vergleichenden Studie anwenden, so müsste man mehr in den Blick nehmen als bloß Familie und Staat. Und man müsste auch noch genauer hinsehen, als es zuvor angedeutet war.

\section{Das erweiterte Gefüge rechtlich verfestigter Solidargemeinschaften}

In der Überschrift dieses Beitrags war die Rede von einem Gefüge von Solidargemeinschaften. Danach hieß es im ersten Abschnitt, dass das Sozialrecht das Neben- und Ineinander unterschiedlicher Bezugsgemeinschaften von Solidarität zu organisieren habe. Betrachtet wurden aber nur zwei Solidargemeinschaften: Familie und Staat. Dabei sind dies nicht die einzigen - nicht mit Blick auf die tatsächlich beobachtbare Realität gemeinschaftlicher Solidarität, und auch nicht mit Blick auf die im Recht vorgesehenen Formen solcher Verbundenheit. Vielmehr finden sich dies- und jenseits der staatlichen Grenzen weitere solcher rechtlich verfestigter Solidargemeinschaften.

Diesen Hintergrund der eingangs vorgestellten Konzeption weiter auszuleuchten, ist Ziel dieser ersten Perspektiverweiterung. Zunächst soll exemplarisch illustriert werden, was mit dem Gefüge rechtlich verfestigter Solidargemeinschaften gemeint ist. In einem zweiten Schritt geht es um die Bedeutung, die diese Perspektiverweiterung für den vorgestellten Ansatz hat.

15 Vgl. unten III. 2. 
Innerhalb der staatlichen Grenzen gibt es eine Fülle von Beispielen. Oft ist es das Sozialrecht im engeren, herkömmlichen Sinne, das diese konstituiert. Man denke nur an die verschiedenen Systeme der Sozialversicherung, die kleinere Gemeinschaften wechselseitiger solidarischer Verpflichtung innerhalb der staatlichen Gemeinschaft konstituieren. Definiert werden diese im Einzelnen über die Bestimmungen zu Versicherungspflicht sowie freiwilliger Versicherung. Oft sind sie darüber hinaus noch unterteilt in regionale, berufsständische, sektorale oder betriebliche Untereinheiten.

$\mathrm{Zu}$ beachten ist ferner, dass auch „die staatliche Gemeinschaft“ in ihren Grenzen keineswegs einheitlich ist, wenn es um solche Beziehungen solidarischer Verpflichtung geht, wie sie in der Sozialversicherung, aber auch im Bereich steuerfinanzierter Sozialleistungen festgeschrieben sind. Schließlich erfassen die einzelnen Regelungskomplexe von Belastung und Begünstigung nicht immer alle Staatsbürger, und vor allem auch nicht ausschließlich diese. Regelmäßig handelt es sich vielmehr um variierende Ausschnitte der Wohnbevölkerung. Überdies ist diese staatliche Gemeinschaft als solche ihrerseits regional unterteilt, und es finden sich in zwar begrenztem, aber doch nicht unerheblichem Maße auch auf Ebene der Länder und Kommunen Formen rechtlich verfestigter Solidarität.

Solche Formen finden sich darüber hinaus auch außerhalb von Steuer- und Sozialrecht im herkömmlichen Sinne. Das Familienrecht als vielleicht wichtigstes Beispiel steht hier ohnehin im Zentrum des Interesses. Aber auch das Arbeitsrecht sieht mitunter solidarische Verpflichtungen zwischen Arbeitnehmer und Arbeitgeber vor, wenn auch gewiss in deutlich enger begrenztem Umfang.

Ferner lassen sich auch jenseits der staatlichen Grenzen rechtliche Substrate solidarischer Verbundenheit identifizieren - jedenfalls in Europa. Zwar mag man die Aussichten auf eine „Sozialunion“, die diesen Namen verdient, durchaus skeptisch beurteilen. ${ }^{16}$ Und auch der Verweis darauf, dass rechtliche Verfestigungen von Solidarität auf supranationaler Ebene bislang höchst indirekt sind, ist vollends berechtigt. Dennoch wird man in den beträchtlichen Transferleistungen, wie sie innerhalb der EU etwa im Rahmen regionaler Strukturförderung 17 stattfinden, und den entsprechenden Modi ihrer Finanzierung Ausprägungen solcher rechtlich fixierter Solidarität erblicken können.

16 Vgl. für eine solche Sicht exemplarisch Zacher, Wird es einen europäischen Sozialstaat geben?, EuR 2002, S. $147 \mathrm{ff}$.

17 Einzelheiten zum EU-Haushalt lassen sich auf jeweils aktuellem Stand einsehen unter http://ec.europa.eu/budget/budget_detail/current_year_de.htm (eingesehen am 25.9.2008). 


\section{b) Die Bedeutung des erweiterten Gefüges für den zuvor vorgestellten Ansatz}

Ein komplexes Gefüge rechtlich verfestigter Solidargemeinschaften, wie es soeben exemplarisch skizziert wurde, wird man in entwickelten Rechtsordnungen regelmäßig vorfinden. Gewiss weist es nicht überall dieselbe Struktur auf. Mit Blick auf den Grad transnationaler Gemeinschaftsbildung stellt Europa, wie bereits angedeutet, ohnehin eine Ausnahme dar. Aber auch mit Blick auf die anderen, innerstaatlichen Gemeinschaften kann man mit Unterschieden zwischen verschiedenen Rechtsordnungen rechnen. Für eine vergleichende Betrachtung lässt sich aus der vorangegangenen Illustration also lediglich der Schluss ziehen, dass man es potentiell mit einer Vielheit von Gemeinschaften zu tun hat. Bei der konkreten Gestalt des Gefüges hingegen wird man mit Unterschieden rechnen müssen.

Überdies ist das Gefüge auch wandelbar. Zuvor war bereits auf jene allmähliche Verlagerung von der Familie hin zur staatlichen Gemeinschaft hingewiesen worden, wie sie sich in manchen Entwicklungen des positiven Rechts manifestiert. Einen ähnlichen Trend könnte man wohl auch in jenem erweiterten Gemeinschaftsgefüge ausmachen, das nun in diesem Abschnitt in den Blick genommen wurde. Auch in diesem komplexeren Gefüge dürfte sich eine allmähliche Verlagerung nachweisen lassen von den kleineren hin zur größeren Gemeinschaft, also regelmäßig zur Nation als der bislang in den meisten Regelungskontexten noch immer größtmöglichen Bezugseinheit für jenes Ziel kollektiver Gleichheitsgenerierung, das zu verfolgen das Sozialrecht nach dem hier zugrunde gelegten Verständnis kennzeichnet. 18

Vor dem Hintergrund der hier vorgenommenen Perspektiverweiterung ist es also nur ein Ausschnitt des Gefüges rechtlich verfestigter Solidargemeinschaften, auf den der zu Beginn des Beitrags vorgestellte Ansatz fokussiert. Das stellt den Ansatz nicht grundsätzlich in Frage. Es kann durchaus sinnvoll sein, sich auf das Wechselspiel einzelner Gemeinschaften zu konzentrieren. Vielmehr wäre sogar umgekehrt fraglich, ob eine umfassende Perspektive, zumal in einer vergleichenden Untersuchung, je praktikabel sein könnte. Aber es ist dennoch von Vorteil, sich des Hintergrundes bewusst zu sein teils, weil die hier herausgegriffenen Gemeinschaften in mancher Hinsicht mit den anderen verwoben sind, teils, weil sich Veränderungen im Verhältnis der betrachteten Gemeinschaften, wie angedeutet, als Teil breiterer Trends innerhalb des Gemeinschaftsgefüges darstellen könnten.

18 Näher zu diesem Trend und seiner Widersprüchlichkeit Graser, Grenzenlose Solidarität?, Rechtsgeschichte 2004, S. $29 \mathrm{ff}$. 


\section{Die Familie als ein (auch) gewillkürtes Verhältnis}

Familiale Beziehungen wurden zuvor pauschal als „zufällige“ behandelt. Auch darin lag eine Verkürzung. Denn das sind sie nicht, jedenfalls nicht alle, und gewiss nicht alle in gleichem Maße. ${ }^{19}$

Ehen beispielsweise werden im Regelfall bewusst und freiwillig eingegangen. Die daraus resultierenden Pflichten lassen sich insofern auf einen Akt zurückführen, mit dem die Partner ihr Schicksal willentlich gestalten. Auch bei den entsprechenden Pflichten gegenüber Nachkommen mag man das so sehen. Zwar nimmt die Annahme nüchterner und vor allem vorausschauender Willensbetätigung hier schon fiktivere Züge an. Aber in ihrer Entstehung sind diese Pflichten keineswegs so zufällig wie jene, die zuvor als Beispiel dienten, also die der Nachkommen gegenüber ihren Eltern.

Diese Elemente autonomer Gestaltung bei der Entstehung familialer Beziehungen beseitigen jedoch die Zufälligkeit der daraus resultierenden Verpflichtungen nur teilweise. Schließlich sind die entstehenden Rechtsverhältnisse grundsätzlich als dauerhafte konzipiert. Und die wechselseitigen Einstandspflichten reduzieren sich keineswegs auf das, was bei deren Begründung vorhersehbar gewesen wäre. Insofern behält die Familie den Charakter einer Schicksalsgemeinschaft, die den Wechselfällen des Lebens - jedenfalls nach Maßgabe der gesetzlichen Regelungen - in solidarischer Verbundenheit ausgesetzt ist. ${ }^{20}$ Dieser Konzeption entspricht es ferner, dass es auch nicht ohne Weiteres möglich ist, sich aus dieser Verbundenheit zu lösen, wobei sich auch hier wieder Differenzierungen anbringen ließen, was die rechtliche Verfestigung der zuvor unterschiedenen Beziehungen innerhalb der Familie betrifft.

Bei näherem Hinsehen ergibt sich also ein differenzierteres Bild. Die „Zufälligkeit“ familialer Bindungen kennt Grade. Reflexe dieser Abstufungen zeigen sich auch in entsprechenden Abstufungen bei der Verantwortungsteilung zwischen den beiden Solidargemeinschaften von Familie und Staat, wie sie im positiven Recht konzipiert ist. In diesem Sinne lassen sich beispielsweise die Unterschiede im Ausmaß der innerfamilialen Unterhaltsverantwortung deuten, nämlich einerseits der Kinder gegenüber ihren Eltern und andererseits umgekehrt der Eltern gegenüber ihren Kindern. Ersterer, also der zufälligere Typus, ist regelmäßig ${ }^{21}$ stärker über den kleinen Rahmen der Familie hinaus kol-

19 Auf diesen Aspekt hat in der Diskussion Dagmar Coester-Waltjen hingewiesen.

20 Vgl. zu diesem Leitbild und dessen verfassungsrechtlichen Grundlagen statt vieler wieder Boecken/Klattenhoff(Fußn. 5), JZ 2006, S. 287 f.

21 Einen Sonderfall erörtern Boecken/Klattenhoff (Fußn. 5), JZ 2006, S. 287. Es geht dabei um die unterhaltsrechtliche Inanspruchnahme erwachsener Kinder zugunsten ihrer pflegebedürftigen Eltern durch den Sozialhilfeträger, die weiter reicht als umgekehrt die Inanspruchnahme von Eltern pflegebedürftiger, erwachsener Kinder. Boecken/Klattenhoff halten diese Privilegierung der Eltern für gerechtfertigt über den Gedanken des Ausgleichs jener Sonderlast, wie sie die Eltern eines Kindes trügen, dessen Pflegebedürftigkeit (Boecken/Klattenhoff sprechen hier von Behinderung) ,,regelmäßig lebenslang" bestehe. 
lektiviert als letzterer. Wie die Zufälligkeit, so kennt also auch das Ausmaß der solidarischen Bindung Grade, und mitunter korrespondiert dies.

Wie im vorigen Abschnitt gilt auch hier, dass diese zusätzliche Differenzierung im Hinblick auf die Zufälligkeit familialer Beziehungen den Ansatz zwar in relevanter Weise erweitert. Schließlich sind Grautöne sichtbar geworden, die es zuvor nicht waren. Grundsätzlich in Frage gestellt ist der Ansatz dadurch aber nicht. Der Blick auf diesen Ausschnitt des Gefüges rechtlich verfestigter Solidargemeinschaften bleibt weiterhin möglich. Und er bleibt auch sinnvoll, zumal auch der zuvor skizzierte Zielkonflikt, innerhalb dessen die Verantwortungsteilung zwischen Familie und Staat zu erfolgen hat, im Grundsatz auch nach dieser Differenzierung fortbesteht.

\section{Die Bedeutung sozialer Dienste}

Als eingangs die drastischen Unterschiede veranschaulicht werden sollten, welche zwischen deutschem und US-amerikanischem Recht hinsichtlich der Rolle bestehen, welche die familiale Solidarität im Verhältnis zur staatlichen spielt, da war ausschließlich von solchen Rechten und Pflichten die Rede, die Vermögenswerte betreffen: Unterhalts- und Pflichtteilsansprüche, Familienleistungen, Steuerpflichten. Auch darin lag eine Verkürzung. Denn das In- und Nebeneinander von Familie und Staat wird nicht allein von solchen Rechtsverhältnissen bestimmt. Vielmehr spielen auch soziale Einrichtungen, Dienste und Interventionsbefugnisse eine wesentliche Rolle. ${ }^{22}$

Besonders deutlich wird dies erneut, wenn man die Perspektive der Kinder einnimmt. Schließlich ist aus dieser Sicht die familiale Eingebundenheit am zufälligsten und mithin auch das Bild der Familie als Hort ungerechtfertigter gesellschaftlicher Ungleichheiten am plausibelsten. Denn in welche Familie man geboren ist, bestimmt nicht nur die materiellen Bedingungen, unter denen man aufwächst, und die Wahrscheinlichkeit, mit der man später einmal für die eigenen Eltern sorgen muss oder umgekehrt von ihnen erbt. Es beeinflusst vielmehr auch die Bildungschancen, die man erhält, ${ }^{23}$ und das Risiko, dass man zum Opfer von Verwahrlosung oder gar Gewalt werden wird. ${ }^{24}$ Und wie im Falle der materiellen Bedingungen, so können zufällige Ungleichheiten auch hin-

22 Auf diesen Aspekt hat in der Diskussion Rainer Pitschas hingewiesen.

23 Siehe dazu exemplarisch für die soziale Herkunft der Studenten Bundesministerium für Bildung und Forschung, Die wirtschaftliche und soziale Lage der Studierenden in der Bundesrepublik Deutschland 2006, 18. Sozialerhebung des Deutschen Studentenwerks, Berlin 2007, S. 117 ff. (S. 133 ff.), http://www.studentenwerke.de/se/2007/Hauptbericht18SE.pdf (eingesehen am 25.8.2008).

24 Vgl. Knost, Auswirkungen von Armut und Arbeitslosigkeit auf die psycho-soziale Entwicklung der Betroffenen, insbesondere der Kinder und Jugendlichen, Dortmund 2000; Pfeiffer/Wetzels/Enzmann, Innerfamiliäre Gewalt gegen Kinder und Jugendliche und ihre Auswirkungen, KFN Forschungsbericht Nr. 80, Hannover 1999, S. 11, 13 ff. 
sichtlich solcher immateriellen Bedingungen wie der soeben genannten abgemildert werden, indem die größere, staatliche Gemeinschaft ausgleichend wirkt.

Die Formen solchen Ausgleichs immaterieller Unterschiede können unterschiedliche sein. Geht es um den Schutz vor Verwahrlosung und Gewalt, könnte ein Ausgleich im Wege der staatlichen Anleitung, Kontrolle, und ggf. Intervention stattfinden. Der Blick wäre mithin zu richten auf Regelungen der Kinder- und Jugendhilfe, des Vormundschaftsrechts etc. Geht es dagegen um den Ausgleich unterschiedlicher Bildungschancen, wären Institutionen wie Kindergarten und Schule und insbesondere deren Zugänglichkeit und Durchlässigkeit maßgeblich.

Bei dieser kursorischen Illustration mag es hier sein Bewenden haben. Die Folgerung ist klar. Wer das Verhältnis von staatlicher und familialer Solidarität im Recht betrachten möchte, sollte sich auch mit (in einem weiten Sinne) sozialen Diensten befassen. Das gilt besonders, wenn man eine solche Betrachtung vor dem Hintergrund der zuvor skizzierten Sicht anstellt, dass die Familie Antipode eines - im Wortsinne - „modernen“ Sozialrechts sein könnte.

Praktisch dürfte der Einbezug der sozialen Dienste die Komplexität einer entsprechenden Untersuchung deutlich steigern. Rechte und Pflichten, die sich auf Vermögenswerte beziehen, lassen sich leichter erfassen und vergleichen. Absehbar ist überdies, dass sich der deutliche Kontrast, den das deutsche und das US-amerikanische Recht in der stark verkürzten Darstellung zuvor boten, in einer solchen differenzierteren Betrachtung relativieren würde. Einwände gegen den Ansatz oder seine Erweiterung sind dies freilich nicht.

\section{Dogmatik und Sozialrechtsvergleich}

Der vorliegende Band versammelt Beiträge, die sich mit Rechtvergleichung und Dogmatik im Sozialrecht befassen sollen. Vergleichend ist mein Beitrag ohne Frage, und auch als sozialrechtlich dürfte er wohl „durchgehen“, jedenfalls wenn man dem zu Anfang begründeten weiten Begriffsverständnis folgt. Von Dogmatik dagegen ist bisher wenig die Rede gewesen. Außerdem war der Ansatz eingangs als ,soziologisch orientiert" bezeichnet worden. Würde er also vielleicht gar nicht in diesen Band gehören? Und wenn doch: Wo kommt die Dogmatik ins Spiel?

\section{Rechtswissenschaftliche Erkenntnisziele}

Die akademische Befassung mit Recht kann mindestens drei verschiedenen Zwecken dienen. Erstens kann sie darauf gerichtet sein, die Rechtsanwendung zu unterstützen, indem sie sich mit der Auslegung des positiven Rechts sowie deren Methoden befasst 
und eine Systematisierung dieses komplexen und sich kontinuierlich wandelnden Stoffes betreibt. Diese (in einem weiten Sinne) dogmatische Dimension dürfte in der rechtswissenschaftlichen Praxis quantitativ die überwiegende sein. Auch ist dies eine Domäne nahezu ausschließlich der Juristen, in die Vertreter anderer Disziplinen kaum einmal vordringen. ${ }^{25}$

Zweitens kann Rechtswissenschaft aber auch darauf zielen, die Regeln für eine funktionstüchtige, zweckmäßige oder auch gerechte Ordnung zu ermitteln. Auch diese (abermals in einem weiten Sinne) steuerungswissenschaftliche Dimension findet in der rechtswissenschaftlichen Praxis einige Verbreitung. ${ }^{26}$ Allerdings können die Juristen diesen Bereich nicht für sich allein beanspruchen, sondern teilen ihn sich mit Vertretern (anderer) normativ orientierter Sozialwissenschaften.

Drittens schließlich kann das Recht aber auch als gesellschaftliches Phänomen und Spiegel einer umfassenderen sozialen Realität betrachtet werden. ${ }^{27}$ In dieser soziologischen oder kulturwissenschaftlichen Ausrichtung wird Rechtswissenschaft am seltensten betrieben. Meist findet man derlei Perspektiven in Arbeiten einer historisch orientierten (Rechts-)Soziologie oder (Rechts-)Anthropologie, wobei die primäre disziplinäre Prägung der in diesem Feld arbeitenden oft nicht eine juristische ist.

\section{Rechtsvergleichende Erkenntnisziele}

Die Zwecke speziell der Rechtvergleichung entsprechen denen ihrer Mutterwissenschaft, der Rechtswissenschaft. Aber die Akzente liegen anders bei der Rechtvergleichung. Gemeinhin sind es hier zwei Ziele, die in der umfangreichen Methodenliteratur besonders hervorgehoben werden:

25 Eine Ausnahme bildet die ,law and economics“-Bewegung - gegenwärtig der vielleicht am weitesten reichende Vorstoß disziplinfremder Personen oder doch jedenfalls Gedanken in den Bereich der dogmatischen Rechtswissenschaft. Eine hierzulande grundlegende Darstellung zu dieser großenteils von den USA ausgehenden Strömung bietet Eidenmüller, Effizienz als Rechtsprinzip: Möglichkeiten und Grenzen der ökonomischen Analyse des Rechts, 3. Aufl. Tübingen 2005.

26 Jedenfalls wenn man diese zweite Herangehensweise strikt im Sinne einer „Gesetzgebungslehre“ versteht, ist eine trennscharfe Abgrenzung von der ersten Herangehensweise nicht ausgeschlossen. Bezieht man dagegen auch den Bereich der Rechtsanwendung und -auslegung, der in praxi unbestreitbar ebenfalls Spielräume für normative Erwägungen lässt, mit in die Betrachtung ein, so verschwimmen die Grenzen zwischen beiden Herangehensweisen.

27 Vgl. besonders eindringlich zu dieser Perspektive Geertz, Local Knowledge - Further Essays in Interpretive Anthropology, 3. Aufl. New York 2000, der Recht betrachtet als ,part of a distinctive manner of imagining the real“ (S. 184) und weiter ausführt ,that in such an approach, law is rejoined to the other great cultural formations of human life - morals, (...) religion, (...) history" (S. 218 f.). 
Das eine wird mit einer klassischen Formel umschrieben als die Anreicherung des „Lösungsvorrates“. ${ }^{28}$ Recht wird hier also steuerungswissenschaftlich betrachtet. Und die vergleichende Betrachtung fremder Rechtsordnungen soll dazu dienen, das Arsenal von zur Verfügung stehenden Regelungsformen zur Lösung gemeinsamer sozialer Problemstellungen zu erweitern.

Das andere Ziel ist prägnant beschrieben in der Formel von der Rechtsvergleichung als Kulturvergleichung. ${ }^{29}$ Nicht der mögliche Transfer von Lösungen von einem System ins andere steht hier also im Vordergrund. Vielmehr soll das Studium von (wie zuvor) unterschiedlichen rechtlichen Regelungen gleicher sozialer Problemlagen Erkenntnisse über die Gesellschaften zu Tage fördern, deren Recht verglichen wird. Es geht also um Erkenntnisziele, die der Soziologie oder Kulturwissenschaft zuzuordnen sind.

Wollte man an dieser Stelle eine Zwischenbilanz ziehen, so wäre festzuhalten, dass die Ziele speziell der Rechtvergleichung jenen entsprechen, die zuvor für die Rechtwissenschaft im Allgemeinen als untergeordnete identifiziert wurden. Die dogmatische Dimension hingegen, also das in praxi Hauptziel der Rechtswissenschaft, schiene demnach für die Rechtsvergleichung keine wesentliche Rolle zu spielen.

Dieser Befund vertrüge sich allerdings kaum mit der Themenstellung der hier dokumentierten Tagung. Denn wie wäre es möglich, dass diese das Verhältnis von Rechtsvergleich und Dogmatik im Sozialrecht ins Zentrum rückt, wenn letztere von allenfalls untergeordneter Bedeutung sein sollte?

\section{Zur (verborgenen) Bedeutung der Dogmatik in der (Sozial-)Rechtsvergleichung}

Tatsächlich erweist sich der Schluss auf die Irrelevanz der Dogmatik im Rechtsvergleich bei näherer Betrachtung als voreilig. Und mehr als das: Gerade mit Blick auf das Werk Zachers und sein Fortwirken ist es im Gegenteil durchaus plausibel, wenn mit der Themenstellung für diesen Band die Bedeutung der Dogmatik in der Sozialrechtsvergleichung den Vertretern dieser Disziplin besonders bewusst gemacht wird.

\section{a) Dogmatische Ziele der Rechtsvergleichung}

Zunächst fällt auf, dass der Rechtsvergleichung mitunter sogar ausdrücklich auch solche Zwecke beigemessen werden, die nach der zuvor getroffenen Einteilung als ge-

$28 \mathrm{Zu}$ dieser Formulierung vgl. etwa Zweigert/Kötz, Einführung in die Rechtsvergleichung, 3. Aufl. Tübingen 1996, S. 14; ebenso Rheinstein/v. Borries, Einführung in die Rechtsvergleichung, 2. Aufl. München 1987, S. 26, beide unter Berufung auf Zitelmann.

29 Vgl. hierzu insbesondere Häberle, Rechtsvergleichung im Kraftfeld des Verfassungsstaates, Berlin 1992; auch Wahl, Verfassungsvergleichung als Kulturvergleichung, in: Ders. (Hrsg.), Verfassungsstaat, Europäisierung, Internationalisierung, Frankfurt a. M. 2003, S. 96 ff. 
nuin dogmatische gelten könnten. Besonders pointiert tritt dies in jener vereinzelt erhobenen Forderung hervor, die Rechtsvergleichung als fünfte in den Kanon klassischer Auslegungsmethoden aufzunehmen. ${ }^{30}$

Plausibilität kann diese Forderung vor allem in jener (Sonder-)Konstellation mehrerer auf gegenseitige Angleichung ausgerichteter Rechtsordnungen beanspruchen, wie man sie derzeit im zusammenwachsenden Europa antrifft. Darüber hinaus lässt sich dieses Postulat aber auch allgemeiner darauf stützen, dass der Rechtsauslegung - wie jeder Interpretation - unweigerlich auch schöpferische Elemente innewohnen. Die Berücksichtigung rechtsvergleichender Erkenntnisse bei der Rechtsauslegung würde sich demnach schlicht als besonderer Anwendungsfall der steuerungswissenschaftlichen Zielsetzung der Rechtsvergleichung darstellen.

$\mathrm{Zu}$ allgemeiner Anerkennung jedoch hat es die Forderung, die Rechtvergleichung allgemein zur Auslegungsmethode zu erheben, noch nicht gebracht. ${ }^{31}$ Und über deren Berechtigung und vor allem Tragweite könnte man im Einzelnen tatsächlich streiten. Doch braucht dies hier nicht näher erörtert zu werden. Denn es handelt sich hierbei keineswegs um die einzige, und wohl auch nicht um die bedeutsamste Funktion, welche der Dogmatik in der Rechtsvergleichung zukommt.

\section{b) Die unverzichtbare (Hilfs-)Funktion der Dogmatik}

Die Rechtsvergleichung, wie zuvor, über eine Klassifikation ihrer Erkenntnisziele zu bestimmen, ist nur eine unter mehreren Möglichkeiten der Annäherung. Die Rolle der Dogmatik kommt auf diesem Wege kaum zum Vorschein. Sichtbar wird deren zentrale Bedeutung dagegen, wenn man nicht auf die Ziele, sondern den Prozess der Vergleichung blickt. Zacher hat diesen Prozess mit dem Dreischritt „erfassen, verstehen, bewerten" zusammengefasst, 32 und schon anhand dieser basalen Formel wird deutlich, in welchen Phasen rechtsvergleichender Studien die Dogmatik jedenfalls Bedeutung erlangt.

Die zuvor genannten Ziele der Rechtvergleichung, unter denen die Dogmatik allenfalls eine untergeordnete Rolle spielt, lassen sich dem letzten Schritt des „Bewertens“

30 Häberle tritt seit langem dafür ein, die Rechtsvergleichung als „fünfte Auslegungsmethode“ zu etablieren. Erst jüngst hat er diese Forderung wieder bekräftigt; vgl. Häberle, Funktionen und Bedeutung der Verfassungsgerichte in vergleichender Perspektive, EuGRZ 2005, S. 685 ff.

31 Zwar kann Häberle, Rechtsvergleichung im Kraftfeld des Verfassungsstaates (Fußn. 29), immerhin mit Blick auf die Verfassungsrechtsprechung einen Trend in diese Richtung dokumentieren. Aber sein rechtsvergleichender Überblick zeugt auch davon, wie weit sein Postulat in den meisten Verfassungsgerichten noch von der Durchsetzung entfernt ist.

32 Vgl. Zacher, Vorfragen zu den Methoden der Sozialrechtsvergleichung, S. 21 ff. (22 f.), in: Ders. (Hrsg.), Methodische Probleme des Sozialrechtsvergleiches, Colloquium der Projektgruppe für Internationales und Vergleichendes Sozialrecht der Max-Planck-Gesellschaft, Tutzing 1976, Berlin 1977. 
zuordnen. Die beiden notwendigen Vorstufen des „Erfassens“ und „Verstehens“ dagegen sind bislang noch nicht erwähnt worden, und es sind diese Phasen, in denen der Dogmatik eine unverzichtbare Funktion zukommt.

Bereits die Erfassung des für den Vergleich relevanten Materials erfordert schließlich eine gezielte Selektion, wie sie ohne die systematisierenden Vorleistungen der Dogmatik kaum denkbar wäre. Ähnlich gilt für das allmähliche Verstehen des Materials, dass es sich ohne Wissen um die systematischen Zusammenhänge und Anwendungsmodi der betrachteten Regeln kaum einstellen kann. Denn wie sollten beispielsweise, bezogen auf den zuvor umrissenen Ansatz, die einschlägigen Rechtsbereiche von Sozial- und Steuerrecht, von Unterhalts- und Erbrecht sowie noch eine Reihe weiterer Regelungsfelder identifiziert werden, und wie sollten ferner die einzelnen Normen in ihrer konkreten Anwendung und Wechselbezüglichkeit verstanden werden, also etwa die Regeln zum Elternunterhalt und ihr Zusammenhang mit jenen des Grundsicherungsgesetzes - kurz: wie sollte das Material erfasst und verstanden werden, wenn nicht auf Grundlage jener Systematik und Methodik, die zu entwickeln und zu pflegen Aufgabe der Rechtsdogmatik ist?

Was immer also die Ziele eines Rechtsvergleichs sein mögen - ob, wie in der zuvor vorgestellten Ausrichtung, das kulturvergleichende (oder weniger hochtrabend: landeskundliche) Interesse im Vordergrund steht, oder ob es konkrete Regelungsziele sind, um die es geht - ohne die Vorleistungen der Dogmatik würden Missverständnisse und Oberflächlichkeit drohen. Der Dogmatik kommt für die Rechtsvergleichung zwar in diesem Sinne nur eine Hilfsfunktion zu. Unverzichtbar ist sie freilich dennoch.

\section{c) Dogmatische Expertise als Proprium der Sozialrechtsvergleichung}

Auch wenn nach alledem die Fortentwicklung der Rechtsdogmatik nicht zu den primären Zielen der Rechtvergleichung zu zählen ist, so ist dogmatische Expertise doch eine notwendige Voraussetzung für das Praktizieren von Rechtsvergleichung. So wenig, wie sich das komplexe und hoch ausdifferenzierte Recht moderner Gesellschaften ohne die entsprechende Expertise anwenden ließe, ebenso wenig dürfte dieses Recht, ohne dass diese Voraussetzung erfüllt wäre, auch im Rahmen vergleichender Betrachtungen je zu verstehen sein - gleich ob dieses Verstehen nun steuerungswissenschaftlich oder kulturvergleichend motiviert ist.

Gewiss können sozialwissenschaftliche Vergleiche auch ohne solche Expertise auskommen. Offenkundig können und werden etwa Wohlfahrtsstaaten, 33 Gesundheitssysteme $^{34}$ oder Aktivierungsmaßnahmen ${ }^{35}$ durchaus ertragreich verglichen, auch ohne

33 Zur Illustration sei hier statt vieler nur das Standardwerk von Esping-Andersen, The Three Worlds of Welfare Capitalism, Princeton 1990, zitiert.

34 Vgl. aus diesem Bereich exemplarisch etwa Immergut, Health Politics: Interests and Institutions in Western Europe, Cambridge 1992. 
dass dabei rechtsdogmatische Kenntnisse einbezogen würden. Allein besetzen kann die (Sozial-) Rechtsvergleichung dieses Feld nicht, ebenso wie die (Sozial-) Rechtswissenschaft sich die Felder steuerungs- oder kulturwissenschaftlicher Betrachtungen mit den Vertretern anderer sozialwissenschaftlicher Disziplinen teilen muss.

Umso sinnvoller freilich ist es vor diesem Hintergrund, wenn sich (Sozial-) Rechtswissenschaft und -vergleichung auf ihr Proprium besinnen, auf ihr Wissen um Systematik und Anwendungsmodalitäten des Rechts, und wenn sie diese dogmatische Expertise in Studien mit einer entsprechenden Ausrichtung und Tiefenschärfe zum Einsatz bringen. So, und vielleicht nur so kann sie ihre Eigenständigkeit innerhalb eines unweigerlich multidisziplinären Diskurses behaupten und rechtfertigen. Als ein solches Monitum zur Besinnung auf die genuin rechtswissenschaftliche Methodik verstehe ich die Themenstellung des vorliegenden Bandes. Und was läge näher, als dies anlässlich einer Tagung, mit der das Werk des Gründungsdirektors eines sozialrechtsvergleichenden Instituts gewürdigt werden soll, den Vertretern dieser Disziplin ins Gedächtnis zu rufen?

Die Alternative zu einer solchen (Rück-) Besinnung auf die rechtswissenschaftliche Methodik wäre ein Rückzug auch bei den Erkenntniszielen auf die alleinige Domäne der Juristen, eine Beschränkung also auf den Bereich rein dogmatischer Fragestellungen. Für die Rechtswissenschaft wäre dies bedauerlich, weil sie sich fruchtbarer Betätigungsfelder begäbe. Für die Rechtsvergleichung könnte es fatal sein, weil sie soziale Relevanz vor allem dann erlangen kann, wenn sie die Dogmatik transzendiert.

Ein Nebenertrag aus Überlegungen dieses letzten Abschnitts wäre, dass der vorliegende Beitrag doch in diesen Band passen könnte - aller soziologischen Orientierung des vorgestellten Ansatzes zum Trotz. Es bleibt zu hoffen, dass der Jubilar diese Einschätzung teilt.

35 Einen Überblick über die umfängliche sozial-, und zwar meist wirtschaftswissenschaftliche Literatur in diesem Bereich bieten etwa Koch/Stephan/Walwei, Workfare: Möglichkeiten und Grenzen, IAB Discussion Paper 17/2005; ebenso Sinn/Meister/Ochel/Werding, Reformkonzepte zur Erhöhung der Beschäftigung im Niedriglohnbereich: Ein Überblick, ifo Schnelldienst 60 (4), 2007, S. 3 ff. Dass freilich eine auch juristisch informierte Perspektive, gerade wenn wie hier die Ebene der Makrovergleiche verlassen ist, zusätzliche Einsichten verheißt, zeigt in diesem Bereich die Arbeit von Handler, Social Citizenship and Workfare in the United States and Western Europe, Cambridge 2004. 


\title{
Sozialstaatsentlastung durch mehr Familiensolidarität
}

\author{
Ursula Köbl
}

I. Öffentliche Familienförderung im Zentrum der Politik - Verstärkung privater Familiensolidarität am Rande

II. Monetäre Anerkennung für private Langzeitpflege und Kleinkindbetreuung 396

III. Ausbau der Familiensolidarität zugunsten von Kindern Überforderungstendenz bei „Patchworkfamilien“

IV. Unterhaltsbeziehungen zwischen erwachsenen Kindern und Eltern 401

V. Ambivalente Tendenzen in Bezug auf nicht (mehr) verheiratete Partner 403

VI. Normativ stabile Familiensolidarität im Erbrecht 407

VII. Schlussbemerkung: Unvermeidlichkeit von Zielkonflikten 411

\section{I. Öffentliche Familienförderung im Zentrum der Politik - Verstärkung privater Familiensolidarität am Rande}

Die Hochkonjunktur von Familienpolitik und Familienförderung sowie ihre ebenso starke und vielstimmige Begleitung durch die Sozialrechtswissenschaft ${ }^{1}$ lassen es nicht sinnvoll erscheinen, in dieser Perspektive verharrend, nun akribisch nach irgend disku-

* Frau stud. jur. Katrin Mysliewic und Herrn stud. jur. Martin Würthner danke ich vielmals für ihre Hilfe.

1 Repräsentativ BMFSFJ (Hrsg.), Familie zwischen Flexibilität und Verlässlichkeit, Siebter Familienbericht, BT-Drs. 16/1360; Tagungsband „Familie und Sozialleistungssystem“, SDSRV 57 (2008), mit Beiträgen von Fuchs, Maximilian, Hase, Friedhelm, Ruland, Franz, Kingreen, Thorsten, Münder, Johannes und Knickrehm, Sabine; Eichenhofer, Eberhard, Ehe und Familie im gegenwärtigen Sozialrecht, VSSR 2008, S. 85; ders., Ehe und Familie in der Sozialrechtsordnung, in: Behrends (Hrsg.), Gesetzgebung, Menschenbild und Sozialmodell im Familien- und Sozialrecht, Berlin 2008; Schwab, Dieter, Familie und Staat, FamRZ 2007, S. 1; Kingreen, Thorsten, Familie als Kategorie des Sozialrechts, JZ 2004, S. 938.

Schlaglichtartig wird die enorme Karriere der sozialstaatlichen Familienthematik deutlich an der erst zwei Jahrzehnte zurückliegenden Darstellung der Entwicklung in: Blüm, Norbert/Zacher, Hans F. (Hrsg.), 40 Jahre Sozialstaat Bundesrepublik Deutschland, 1989 (Zacher, Hans F., S. 48, 84, 100; Kaufmann, Franz Xaver, S. 547-560); Zacher, Hans F., Ehe und Familie in der Sozialrechtsordnung, in: Fiedler (Hrsg.), Verfassungsrecht und Völkerrecht, Gedächtnisschrift für Wilhelm Geck, Köln u. a. 1989, S. 955; ders., Abhandlungen zum Sozialrecht, Heidelberg 1993, S. 555. 